\title{
Flexibility in Migratory Behaviour of a Flight Generalist: Migrate Day and Night, Sprinting Across Ecological Barriers
}

Lina Lopez-Ricaurte ( $\square$ lina@ebd.csic.es )

Estación Biológica de Doñana

Wouter Vansteelant

Estación Biológica de Doñana

Jesús Hernández-Pliego

Freelance, Independent Researcher

Daniel García-Silveira

Estación Biológica de Doñana

Ana Bermejo

SEO/BirdLife

Susana Casado

Terra Naturalis

Jacopo Cecere

Istituto Superiore per la Protezione e la Ricerca Ambientale

Javier de la Puente

SEO/BirdLife

Fernando Garcés-Toledano

GREFA

Juan Martínez-Dalmau

GREFA

Alfredo Ortega

Terra Naturalis

Beatriz Rodríguez-Moreno

GREFA

Diego Rubolini

University of Milan

Maurizio Sarà

University of Palermo

Javier Bustamante

Estación Biológica de Doñana 


\section{Research Article}

Keywords: movement patterns, migration research, landscape heterogeneity

Posted Date: December 28th, 2020

DOl: https://doi.org/10.21203/rs.3.rs-130082/v1

License: (c) (i) This work is licensed under a Creative Commons Attribution 4.0 International License. Read Full License 


\section{Abstract}

Understanding what factors drive variation in movement patterns is a key challenge in current migration research. Environmental drivers such as landscape heterogeneity and weather strongly affect birds' migration influencing daily travel schedules and flight speed. For strictly thermal-soaring migrants, typically large birds, weather explains most seasonal and regional differences in speed. In contrast, smaller-sized flight generalists, which alternate between soaring and flapping flight, may be less dependent on weather and thus more likely to be strongly influenced by landscape in daily travel schedules and internal drivers, such as sex. We GPS-tracked the migration performance of 70 lesser kestrels (Falco naumanni), to estimate the relative importance of environmental (wind and landscape), internal (e.g. sex) and seasonal drivers and to what extend do they explain variation in migratory performance, namely speed, distance, and travelling time. We found that tailwind strength explained most of the seasonal difference in migratory parameters. In both seasons lesser kestrels sprinted across ecological barriers and frequently extended migration into the night, while travelling at a slower pace and mainly during the day when not flying over barriers. Our results highlighted that environmental factors far outweighed internal and other seasonal drivers in explaining variation in migration performance of a flight generalist, despite the ability to switch between flight-modes.

\section{Introduction}

The ability to fly gives birds the unique capacity to perform fast seasonal movements up to thousands of kilometres a year across heterogeneous landscapes and ecological barriers ${ }^{1}$. The way migrants undertake this complex journey often shows great flexibility in behaviour ${ }^{2}$. That flexibility is governed by an interplay between (1) internal drivers, such as motion capacity (dependent on, for example, wing morphology), orientation ability, and the individuals' age, sex and/or reproductive state that shape the internal motivation to move $^{3}$; and (2) environmental drivers such as weather conditions and landscape structure that influences connectivity and creates the so-called ecological barriers $3,4,5$. Variation in behaviour is also affected by differences between adults and juveniles and/or males and females through, for example, reproductive advantages associated with early arrivals of adult males to establish

territories in pre-breeding migration $6,7,8$. Weather conditions of a landscape vary within the daily cycle and among seasons influencing migratory behaviour, e.g. flight-mode (soaring, flapping) or foraging patterns $9,10,11$. However, understanding the relative contributions of such environmental and internal drivers to variation in migratory behaviour is often hampered by the lack of high-resolution tracking data for representative samples of individuals ${ }^{7,11}$.

Studies that took account of the interplay of environmental and internal drivers in shaping migratory performance, which is most commonly measured via metrics such as the ground speed, distance travelled, duration of stopovers and route straightness ${ }^{12,13,14}$, present a bias towards large soaring birds, due to their fairly greater size and thus ability to carry large tracking devices. Studies on these species have demonstrated that variation in weather (e.g. winds, turbulence, and thermal or orographic updrafts) 
is often the prevailing factor explaining performance patterns, such as seasonal and regional differences in hourly and daily migration speeds ${ }^{10,12}$. For example, turkey vultures (Cathartes aura) achieved faster speeds and travelled more hours each day during the pre-breeding compared to the post-breeding migration, because thermal uplifts were stronger during the pre-breeding migration ${ }^{15}$. The Oriental honey buzzards (Pernis ptilorhynchus) traverse ecological barriers (East China Sea) during the post-breeding migration when supportive winds are available and detour them during the pre-breeding migration when weather conditions are less favourable for soaring flight ${ }^{16}$. Age and experience are important factors mediating the response to weather in several species which are more prone to soaring flight during migration (e.g. golden eagle, Aquila chrysaetos ${ }^{17}$; osprey, Pandion haliaetus ${ }^{18}$ ). Yet, sex differences tend to explain little variation in travel speeds of soaring migrants ${ }^{19,20}$.

We still know little about the relative importance of environmental, internal and other seasonal drivers in shaping the migratory performance of smaller, non-obligate soaring-gliding migrants ${ }^{21}$. This is an important bias in current migration research because smaller birds are more abundant and, unlike large soaring birds, can use different flight modes, including obligate-flapping passerines and waders as well as the so-called flight-generalists (swallows, bee-eaters, falcons, hawks and harriers), which are capable of covering long distances using both flapping and soaring flight ${ }^{22,23,24}$. Studies on small birds demonstrated seasonal differences in migratory strategies, such as a time-minimising behaviour during the pre-breeding compared to the post-breeding migration ${ }^{11}$. For example, a tracking radar study of longdistance migrant passerines found faster mean airspeeds (the bird's flight speed relative to the surrounding air) during the pre-breeding compared to the post-breeding migration after accounting for seasonal differences in wind and body mass ${ }^{8}$. These findings suggested a greater seasonal motivation to reduce migration time during the pre-breeding migration due to an expected competition at the breeding areas for nests and mates ${ }^{8}$. The ability of flight-generalists to switch between flight-modes gives them more flexibility to cope with weather conditions and thus may make the role of internal and other seasonal drivers more predominant in shaping migratory decisions, compared to obligate-soaring and obligate-flapping birds ${ }^{21}$.

Flight-generalists migrants are capable of flapping flights that extend daily travel schedules (i.e. how migrants shape their daily travels ${ }^{24}$ ) into the night when thermal updrafts are not available ${ }^{25}$. They typically also achieve higher speeds during nocturnal than diurnal migration, enabling them to cross ecological barriers in non-stop flights ("sprints") ${ }^{26}$. For example, the Amur falcon (Falco amurensis) undertakes the longest non-stop water crossing of any bird of prey studied so far, taking 3-4 days to cross the Indian Ocean, from India to East Africa (ca. 3000-4000 km) flying day and night ${ }^{22}$. On the other hand, birds that invest energy in flapping flight also have to recover that energy by foraging, which they may do prior to or after completing the migration, and often also during migration by (a) making stopovers ${ }^{4}$ or (b) intermittent diurnal fly-forage, i.e. combination of foraging and flying in the migratory direction ${ }^{27,28}$. Studies on African-Eurasian migrants like Eurasian hobbies (Falco subbuteo) and Eleonora's falcons (Falco eleonorae) typically revealed significant seasonal variation in travel speed 
between regions, with fast and long flights over barriers and slower and shorter daily flights over nonbarriers ${ }^{28}$. Studies on Eleonora's falcon indicated that when age, landscape and wind conditions are simultaneously analysed, the type of landscape over which they migrate is the most influencing factor on migration speed, likely due to birds alternating between sprinting flights and slow fly-and-forage migration ${ }^{29}$. However, the interplay between such habitat-dependent time schedules with weather conditions and seasonal and sex-specific differences in shaping migratory flight performance in flight generalists is still being resolved.

We studied the migratory behaviour of a flight generalist, the lesser kestrel (Falco naumanni), a smallsized insectivorous trans-Saharan falcon with reversed size dimorphism. During foraging trips ${ }^{30}$ and occasionally on migration ${ }^{22}$, lesser kestrels are known to soar, especially during periods of intense solar radiation. Additionally, their wings have a relatively high aspect ratio, suitable for long bouts of highspeed flapping flight en route ${ }^{31}$, and thus are capable of extending their daily travel schedule into the night ${ }^{32,33}$. Migration of this species has been mostly studied using geolocators ${ }^{34,35}$, and satellite telemetry ${ }^{32,33}$, shedding light on departure and arrival dates, routes, and non-breeding areas. Sarà et al. (2019) revealed that European-breeding lesser kestrel migrates to African non-breeding areas on a broad front across ecological barriers, instead of concentrating at bottlenecks as many soaring migrants do. Moreover, it has been reported that lesser kestrels migrate faster during the post-breeding than the prebreeding migration, although the mechanisms behind these performance patterns have yet to be studied in detail.

Here we computed travel parameters at coarse (trip), intermediate (daily), and fine (hourly) temporal scales. At the trip scale, we analysed seasonal and sex-specific patterns in migratory performance (i.e. mean trip duration, the proportion of travelling/non-travelling days, mean travelling speed and mean route straightness). At the daily scale, we evaluated patterns in performance (i.e. mean daily speed, mean daily distance and mean travelling time) between landscapes (i.e. ecological barriers vs. non-barriers). At the hourly scale, we further assessed spatiotemporal patterns in hourly performance (i.e. ground speeds) of males and females for both post- and pre-breeding migration, specifically comparing barriers vs. nonbarriers and diurnal and nocturnal flight segments. We also analysed diurnal and nocturnal performance when flying over barriers and non-barriers in relation to weather conditions along the kestrels' routes (i.e. tailwind strength, absolute crosswind strength and boundary layer height; BLH hereafter), accounting for sex and season-specific responses.

To assess which factors drive migratory variation in the lesser kestrel, we first described behavioural patterns by investigating seasonal and sex-specific differences in performance between landscapes, diurnal and nocturnal flights. Then we uncovered mechanisms by disentangling the effect of external (wind, landscape), internal (sex) and other seasonal drivers. It is well established that wind conditions have a significant influence in migration ground speeds ${ }^{1,22}$. We thereby expected a positive effect of tailwind strength will explain a large part of the variation in travel speed along the kestrels' flight path. Accounting for these effects in our models should allow us to assess whether faster post-breeding 
migrations are a consequence of seasonal wind regimes, and whether such wind effects are masking sex-specific and other seasonal drivers, e.g. time-selected behaviour, particularly strong in males ${ }^{36}$, during the pre-breeding migration arising when there is a gain from arriving early at their destinations, which may be challenging to disentangle $e^{6,11,12}$.

Based on the expectations that: a) territory acquisition and competitions for mates increase selective pressure for early arrivals in the breeding relative to the non-breeding areas ${ }^{8,11}$; $b$ ) flight generalists species are less restricted by environmental (and especially weather) conditions compared to obligate soaring-gliding migrants, we hypothesised that models accounting for weather effects will show (1) significantly higher migratory performance (i.e. faster speed, longer daily distance and more travelling hours per day) during the pre-breeding than during the post-breeding migration; (2) significantly higher migratory performance for the smaller males than for the larger females, because flapping is theoretically less costly for the former ${ }^{37}$ and competition for securing a high-quality territory is weaker in the latter ${ }^{38}$. Furthermore, we hypothesised that (3) lesser kestrels migrate faster when flying over ecological barriers (Mediterranean Sea and Sahara Desert) than over non-barriers areas to reduce the time spent in harsh environments where there are few or no resting/drinking/feeding opportunities ${ }^{24,28,39}$. Accordingly, we expected that individuals will perform faster travel speed, larger daily beeline distance, and more extended daily travel schedules, including the use of nocturnal migration when flying over ecological barriers vs. non-barriers.

\section{Results}

\section{Trip scale: Seasonal and sex-specific performance}

We obtained GPS data for 141 (75 post-breeding and 66 pre-breeding) complete migratory tracks from 70 individuals (Fig. 1). Consistent with previous findings, our data showed that lesser kestrels migration was significantly shorter and birds spent fewer days travelling during the post-breeding than during the prebreeding migration (mean duration \pm SE: $8.62 \pm 0.44$ days during the post-breeding vs. $15.62 \pm 1.04$ days during the pre-breeding, $\mathrm{W}=1244, \mathrm{p} \leq 0.05$; mean travelling days $\pm \mathrm{SE}: 7.66 \pm 0.29$ days during the postbreeding vs. $9.46 \pm 0.41$ days during the pre-breeding migration, $W=1722, p \leq 0.05$ ). Birds progressed significantly faster during the post-breeding than during the pre-breeding migration $\left(t_{139}=3.88, p \leq 0.05\right)$, with a mean of $405 \pm 14.33 \mathrm{~km} /$ day vs. $331.03 \pm 12.21 \mathrm{~km} /$ day, respectively (excluding non-travelling days). They made significantly fewer stopovers (non-travelling days) during the post-breeding than during the pre-breeding migration ( $W=1111, p \leq 0.05$ ), with a mean of $1 \pm 0.23$ days vs. $6 \pm 0.78$ days, and followed straighter paths (post-breeding mean straightness index was $0.86 \pm 0.01$ vs. $0.76 \pm 0.01$ during the pre-breeding migration, $W=3912, p \leq 0.05$ ). Observed seasonal patterns were similar between sexes (Fig. 2 and Supplementary Table S1).

\section{Daily scale: barrier-effects on migration performance}


Using data at the daily scale (783 bird-migration days from sunrise to sunrise of the next day), we observed statistically significant differences of mean daily ground speed $\left(F_{1,782}=9.23, p \leq 0.01\right)$, beeline distance $\left(F_{1,782}=16.82, p \leq 0.001\right)$ and travelling time $(z=6.01, p \leq 0.001)$ when flying over barrier vs. non-barrier areas. Lesser kestrels travelled on average $4.5 \mathrm{~km} / \mathrm{h}$ faster, covered $118 \mathrm{~km}$ longer daily distance and flew 0.20 hours more per day when flying over barriers than over non-barriers (Table 1 ).

Table 1

Models for mean daily speed, mean beeline distance and mean travelling time of lesser kestrel accounting for the effect of landscape (when flying over barriers vs. non-barriers) as estimated by LMMs and GLMM (with Poisson error distribution and log link function) accounting for ID as a random effect. Estimates, standard errors $( \pm S E)$ and variation explained $\left(R^{2}\right.$ marginal $)$ are given for each model. $(*=p \leq$ $0.05, * \star=p \leq 0.01, \star \star \star=p \leq 0.001)$.

\begin{tabular}{|c|c|c|c|c|c|c|}
\hline Response & Model & Predictor & Estimate & SE & $t / z$ & $\mathrm{R}^{2}{ }_{\text {mar }}$ \\
\hline \multirow{2}{*}{$\begin{array}{l}\text { Mean daily speed } \\
(\mathrm{km} / \mathrm{h})\end{array}$} & \multirow{4}{*}{$\begin{array}{l}\mathrm{n}=783 \\
\text { individuals }=45\end{array}$} & Intercept & 23.18 & 0.72 & 31.81 & 0.01 \\
\hline & & $\begin{array}{l}\text { Over } \\
\text { barriers }\end{array}$ & 4.58 & 1.51 & $3.03^{\star \star}$ & \\
\hline \multirow{2}{*}{$\begin{array}{l}\text { Mean daily beeline } \\
\text { distance }(\mathrm{km})\end{array}$} & & Intercept & 276.77 & 12.59 & 21.99 & 0.02 \\
\hline & & $\begin{array}{l}\text { Over } \\
\text { barriers }\end{array}$ & 118.93 & 29.00 & 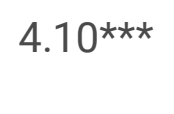 & \\
\hline \multirow[t]{2}{*}{ Mean travelling time $(h)$} & & Intercept & 2.27 & 0.02 & 77.75 & 0.02 \\
\hline & & $\begin{array}{l}\text { Over } \\
\text { barriers }\end{array}$ & 0.20 & 0.03 & $6.01^{\star \star \star}$ & \\
\hline
\end{tabular}

\section{Daily scale: seasonal, sex-specific and external drivers on performance}

As lesser kestrel migration behaviour differed significantly when flying over barriers and non-barriers, we built separate models at the daily scale (see Supplementary Note for details). We first included seasonal and sex-specific factors (including their interaction) as predictors (simple model hereafter). Second, we added wind variables and accounted for intensity of nocturnal travel to explore any seasonal and sexspecific pattern not accounted for by weather conditions encountered en route (complex model hereafter) (Table 2 and Supplementary Table S2). Overall, simple models accounted for relative low variation in daily performance and did not improve model fit (AIC) compared to a more complex model (Table 2). Seasonal daily model estimates during the pre-breeding migration were significantly lower than during the post-breeding migration (Table 2). We did not find significant differences in daily performance between sexes. 
Table 2

Comparisons between simple models for mean daily speed, beeline distance and travelling time accounting only for the interaction effect season:sex vs. more complex final models accounting for the effects of season:sex, nocturnal travel and weather (tailwinds, crosswinds and BLH). We used GLMMs with Gaussian errors for speed and distance and Poisson errors and log link function for travelling time. Model estimates are given in SD units (organised from higher to lower relative importance). Significance tests were performed using ANOVA Wald chi-square test. Season and sex factors did not improve model

fit (AIC) compared to more complex models, neither increased the variation explained $\left(R_{\text {marginal }}^{2}\right)$. All models included individual identity (ID) as a random effect. ( $\left.{ }^{*}=p \leq 0.05, * \star=p \leq 0.01, * \star \star=p \leq 0.001\right)$.

\begin{tabular}{|c|c|c|c|c|c|c|c|}
\hline Response & Model & Predictor & Estimate & SE & $t / z$ & AIC & $\mathrm{R}_{\text {mar }}^{2}$ \\
\hline \multirow{17}{*}{$\begin{array}{l}\text { Daily } \\
\text { speed } \\
(\mathrm{km} / \mathrm{h})\end{array}$} & Over barriers & Intercept & 0.97 & 0.25 & 7.78 & \multirow[t]{4}{*}{307.6} & \multirow[t]{4}{*}{0.10} \\
\hline & \multirow{3}{*}{$\begin{array}{l}\text { Simple model } \\
\text { (Season:Sex) } \\
(\mathrm{n}=100, \\
\text { individuals = } \\
32)\end{array}$} & \multirow{2}{*}{$\begin{array}{l}\text { Season(Pre- } \\
\text { breeding) }\end{array}$} & -0.51 & 0.31 & $-1.67 *$ & & \\
\hline & & & -0.30 & 0.42 & -0.72 & & \\
\hline & & Season:Sex & -0.16 & 0.47 & -0.33 & & \\
\hline & \multirow{4}{*}{$\begin{array}{l}\text { Complex final } \\
\text { model } \\
\text { (Internal + } \\
\text { Weather) }\end{array}$} & Intercept & 0.03 & 0.10 & 0.29 & \multirow[t]{4}{*}{257.5} & \multirow[t]{4}{*}{0.47} \\
\hline & & \multirow{2}{*}{$\begin{array}{l}\text { Nocturnal } \\
\text { travel } \\
\text { fraction }\end{array}$} & 0.51 & 0.08 & $6.40 * \star \star$ & & \\
\hline & & & 0.36 & 0.06 & $5.31^{\star \star \star}$ & & \\
\hline & & Tailwind & & & & & \\
\hline & \multirow{2}{*}{$\begin{array}{l}\text { Over non- } \\
\text { barriers }\end{array}$} & Intercept & 0.05 & 0.07 & 0.63 & \multirow[t]{4}{*}{1878.2} & \multirow[t]{4}{*}{0.03} \\
\hline & & Season:Sex & -0.37 & 0.15 & $-2.44^{\star}$ & & \\
\hline & $\begin{array}{l}\text { Simple model } \\
\text { (Season:Sex) }\end{array}$ & Sex(Male) & 0.22 & 0.12 & 1.82 & & \\
\hline & $\begin{array}{l}(\mathrm{n}=683 \\
\text { individuals = } \\
45)\end{array}$ & $\begin{array}{l}\text { Season(Pre- } \\
\text { breeding) }\end{array}$ & -0.13 & 0.10 & $-1.31^{\star \star \star}$ & & \\
\hline & \multirow{5}{*}{$\begin{array}{l}\text { Complex final } \\
\text { model } \\
\text { (Internal + } \\
\text { Weather) }\end{array}$} & Intercept & -0.12 & 0.04 & -2.76 & \multirow[t]{5}{*}{1497.7} & \multirow[t]{5}{*}{0.44} \\
\hline & & \multirow{4}{*}{$\begin{array}{l}\text { Nocturnal } \\
\text { travel } \\
\text { fraction } \\
\text { Tailwind } \\
\text { Crosswind }\end{array}$} & 0.42 & 0.03 & $13.61^{\star \star *}$ & & \\
\hline & & & 0.33 & 0.03 & $10.91 * \star *$ & & \\
\hline & & & 0.19 & 0.04 & $4.47^{\star \star \star}$ & & \\
\hline & & & & & & & \\
\hline \multirow{4}{*}{$\begin{array}{l}\text { Daily } \\
\text { beeline } \\
\text { distance } \\
(\mathrm{km})\end{array}$} & Over barriers & Intercept & 1.03 & 0.27 & 3.71 & \multirow[t]{4}{*}{317.2} & \multirow[t]{4}{*}{0.05} \\
\hline & Simple model & Season(Pre- & -0.62 & 0.32 & $-1.92^{*}$ & & \\
\hline & \multirow{2}{*}{$\begin{array}{l}(\mathrm{n}=100 \\
\text { individuals = } \\
32)\end{array}$} & \multirow{2}{*}{$\begin{array}{l}\text { Sex(Male) } \\
\text { Season:Sex }\end{array}$} & -0.10 & 0.46 & -0.23 & & \\
\hline & & & 0.05 & 0.50 & 0.11 & & \\
\hline
\end{tabular}




\begin{tabular}{|c|c|c|c|c|c|c|c|}
\hline Response & Model & Predictor & Estimate & SE & $t / z$ & AIC & $\mathrm{R}_{\text {mar }}^{2}$ \\
\hline & \multirow{5}{*}{$\begin{array}{l}\text { Complex final } \\
\text { model } \\
\text { (Internal + } \\
\text { Weather) }\end{array}$} & Intercept & 0.05 & 0.05 & 0.95 & \multirow[t]{5}{*}{164.2} & \multirow[t]{5}{*}{0.81} \\
\hline & & \multirow{2}{*}{$\begin{array}{l}\text { Nocturnal } \\
\text { travelling } \\
\text { hours }\end{array}$} & 0.65 & 0.05 & $12.49 * \star \star$ & & \\
\hline & & & 0.30 & 0.05 & $5.42^{\star \star \star}$ & & \\
\hline & & $\begin{array}{l}\text { Diurnal } \\
\text { travelling } \\
\text { hours }\end{array}$ & 0.24 & 0.04 & $5.95^{\star \star \star}$ & & \\
\hline & & \multicolumn{4}{|l|}{ Tailwind } & & \\
\hline & Over non- & Intercept & 0.02 & 0.07 & 0.31 & \multirow[t]{4}{*}{1863.7} & \multirow[t]{4}{*}{0.03} \\
\hline & \multirow{2}{*}{$\begin{array}{l}\text { Simple model } \\
\text { (Season:Sex) }\end{array}$} & Sex(Male) & 0.27 & 0.11 & 2.32 & & \\
\hline & & Season:Sex & -0.26 & 0.14 & -1.75 & & \\
\hline & $\begin{array}{l}(\mathrm{n}=683 \\
\text { individuals = } \\
5)\end{array}$ & $\begin{array}{l}\text { Season(Pre- } \\
\text { breeding) }\end{array}$ & -0.21 & 0.09 & 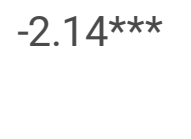 & & \\
\hline & \multirow{7}{*}{$\begin{array}{l}\text { Complex final } \\
\text { model } \\
\text { (Internal + } \\
\text { Weather) }\end{array}$} & Intercept & -0.03 & 0.02 & -1.45 & \multirow[t]{7}{*}{470.5} & \multirow[t]{7}{*}{0.87} \\
\hline & & \multirow{2}{*}{$\begin{array}{l}\text { Nocturnal } \\
\text { travelling } \\
\text { hours }\end{array}$} & 0.66 & 0.01 & $43.07 \star \star \star$ & & \\
\hline & & & 0.28 & 0.01 & $19.68^{\star \star \star}$ & & \\
\hline & & \multirow{2}{*}{$\begin{array}{l}\text { Diurnal } \\
\text { travelling } \\
\text { hours }\end{array}$} & 0.23 & 0.01 & $14.97 * \star \star$ & & \\
\hline & & & 0.05 & 0.02 & $2.03 *$ & & \\
\hline & & Tailwind & & & & & \\
\hline & & $\begin{array}{l}\text { Season(Pre- } \\
\text { breeding) }\end{array}$ & & & & & \\
\hline \multirow{8}{*}{$\begin{array}{l}\text { Daily } \\
\text { travelling } \\
\text { time (h) }\end{array}$} & Over barriers & Intercept & 2.78 & 0.11 & 24.48 & \multirow[t]{4}{*}{753.9} & \multirow[t]{4}{*}{0.06} \\
\hline & Simple model & Season(Pre- & -0.33 & 0.10 & $-3.27 * \star$ & & \\
\hline & \multirow{2}{*}{$\begin{array}{l}(n=100 \\
\text { individuals = } \\
32)\end{array}$} & \multirow{2}{*}{$\begin{array}{l}\text { Season:Sex } \\
\text { Sex(Male) }\end{array}$} & 0.20 & 0.15 & 1.30 & & \\
\hline & & & -0.12 & 0.18 & -0.69 & & \\
\hline & \multirow{4}{*}{$\begin{array}{l}\text { Complex final } \\
\text { model } \\
\text { (Internal + } \\
\text { Weather) }\end{array}$} & Intercept & 3.22 & 0.14 & 22.23 & \multirow[t]{4}{*}{713.3} & \multirow[t]{4}{*}{0.27} \\
\hline & & BLH & -0.57 & 0.10 & $-5.28^{\star \star \star}$ & & \\
\hline & & \multirow{2}{*}{$\begin{array}{l}\text { Season(Pre- } \\
\text { breeding) }\end{array}$} & -0.48 & 0.08 & $-5.71^{\star \star \star \star}$ & & \\
\hline & & & 0.02 & 0.01 & $3.85^{\star \star \star}$ & & \\
\hline
\end{tabular}




\begin{tabular}{|c|c|c|c|c|c|c|c|}
\hline Response & Model & Predictor & Estimate & SE & $t / z$ & AIC & $\mathbf{R}_{\text {mar }}^{2}$ \\
\hline & Over non- & Intercept & 2.31 & 0.04 & 47.34 & \multirow[t]{4}{*}{4230.3} & \multirow[t]{4}{*}{0.02} \\
\hline & Simnle model & Season(Pre- & -0.16 & 0.06 & $-2.62^{\star \star \star}$ & & \\
\hline & (Season:Sex) & \multirow{2}{*}{ Sex(Male) } & 0.15 & 0.07 & 1.97 & & \\
\hline & $\begin{array}{l}(n=683 \\
\text { individuals = } \\
45)\end{array}$ & & -0.10 & 0.09 & -1.10 & & \\
\hline & \multirow{5}{*}{$\begin{array}{l}\text { Complex final } \\
\text { model } \\
\text { (Internal + } \\
\text { Weather) }\end{array}$} & Intercept & 2.25 & 0.07 & 31.39 & \multirow[t]{5}{*}{4050.9} & \multirow[t]{5}{*}{0.22} \\
\hline & & Season(Pre- & -0.25 & 0.04 & $-5.12^{\star \star \star}$ & & \\
\hline & & & -0.16 & 0.06 & $-2.61 * \star$ & & \\
\hline & & & 0.04 & 0.01 & $10.84^{\star \star \star} 7.11^{\star \star \star}$ & & \\
\hline & & Tailwind & 0.02 & 0.01 & & & \\
\hline
\end{tabular}

The final complex model for mean daily speed when flying over barriers and non-barriers accounted for $47 \%$ and $44 \%\left(R^{2}\right.$ marginal $)$ of variation, respectively (Table 2$)$. When flying over both landscapes nocturnal travel fraction and tailwind had a considerable positive effect (Table 2), and this was consistent among both seasons (see Supplementary Figure S1). Over non-barriers we found a positive influence of crosswind strength on mean daily ground speed (Table 2). Season and sex were not selected as significant predictors (Table 2). Over non-barriers, we found that after accounting for wind effect, there was a marginally significant effect of the interaction between season and sex, indicating that the speed difference between males and females during flights over non-barriers was slightly smaller during the prebreeding than during the post-breeding migration (see Supplementary Table 2S).

For daily beeline distance, the final complex models over barriers and non-barriers predicted $81 \%$ and $87 \%$ $\left(R^{2}\right.$ marginal $)$ of all the variation, respectively. When flying over both landscapes nocturnal travelling hours had the strongest positive effects (Table 2). Moreover, we found a positive effect of tailwind, with birds flying farther with stronger mean daily tailwind, although the effect of tailwind strength on daily distance was weaker over barriers during the pre-breeding migration (see Supplementary Figure S1). Mean crosswind and mean BLH were relatively less influential on the daily beeline distance (see Supplementary Table S2). In addition, after accounting for wind effect, individuals displayed slight seasonal differences in daily beeline distance, travelling farther during the pre-breeding relative to post-breeding migration over non-barriers (Table 2). We did not detect an interaction effect between seasons and sexes (see Supplementary Table S2).

The final complex model for daily travelling time over barriers and non-barriers explained $27 \%$ and $22 \%$ $\left(\mathrm{R}^{2}\right.$ marginal $)$ of the variation, respectively. Weather variables best explained the variation in daily travelling 
time when flying over barriers and non-barriers (Table 2). When flying over both landscapes, mean daily travelling time of lesser kestrels was negatively associated with mean BLH and positively associated with absolute crosswind strength (Table 2). We also found substantial seasonal differences after accounting for wind effects, i.e. birds travelled for more hours per day during post-breeding relative to the prebreeding migration when flying over barrier and non-barrier areas (Table 2). We did not detect any significant interaction effect between season and sex (see Supplementary Table S2).

\section{Hourly scale: Diurnal vs. nocturnal migration and barrier- effects on performance}

Our analyses on hourly performance matched the behavioural patterns we observed at the daily scale, with lesser kestrels showing a landscape-dependent propensity for sprinting during nocturnal flights across barriers (Fig. 3 and Supplementary Table S3.a). Based on hourly travel segments (12,252 segments where speed $\geq 5 \mathrm{~km} / \mathrm{h}$ ) lesser kestrels achieved significantly higher hourly speeds at night than during the day $\left(F_{1,12250}=994.91, p \leq 0.001\right)$ and when flying over barriers vs. non-barriers $\left(F_{1,12237}=\right.$ $256.29, p \leq 0.001$ ). Birds travelled on average $6.26 \mathrm{~km} / \mathrm{h}$ faster when flying over barriers than over nonbarriers and $13.12 \mathrm{~km} / \mathrm{h}$ faster during nocturnal than during diurnal flights (Table 3 ).

Table 3

LMM used to model diurnal and nocturnal hourly speeds $(\mathrm{km} / \mathrm{h})$ when flying over barriers vs. non-barriers of lesser kestrels accounting for ID as a random effect. Estimate and standard errors ( $\pm \mathrm{SE})$ and variation explained $\left(\mathrm{R}^{2}\right.$ mar $)$ are given. $(*=p$ $\leq 0.05, * \star=p \leq 0.01, * \star *=p \leq 0.001)$.

\begin{tabular}{|c|c|c|c|c|c|}
\hline Model & Predictor & Estimate & SE & $\mathrm{t}$ & $\mathrm{R}_{\text {mar }}^{2}$ \\
\hline \multirow[t]{3}{*}{$(n=12,252 ;$ individuals $=70)$} & Intercept & 25.61 & 0.41 & 61.49 & 0.15 \\
\hline & Night & 13.12 & 0.33 & $38.70 * \star \star$ & \\
\hline & Barrier & 6.26 & 0.33 & $18.82^{\star \star \star}$ & \\
\hline
\end{tabular}

Faster nocturnal speeds were observed inside and outside barriers, in both seasons, and for both sexes (Fig. 3 and Supplementary Table S3.b). The distribution of travelling and non-travelling segments was significantly different between seasons (post-breeding vs. pre-breeding: $\chi^{2}=840.63 ; D F=1 ; p \leq 0.05$ ) and between landscapes (barrier vs. non-barrier: $\chi^{2}=658.41$; $D F=1 ; p \leq 0.05$ ), but it was not significantly different between sexes (females vs. males: $\chi^{2}=2.79 ; D F=1 ; p=0.09$ ). Most nocturnal migration occurred when flying over barriers and more frequently during the post-breeding than during the prebreeding migration (see Supplementary Figure S2).

\section{Hourly scale: seasonal, sex-specific and external drivers on performance}


We built separate models across four possible combinations of barrier vs. non-barrier and diurnal vs. nocturnal migration, and test for differences in behavioural patterns following the same procedure outlined above. The simple models accounted for less than $3 \%\left(\mathrm{R}^{2}\right.$ marginal $)$ of the variation in mean ground speeds and did not improve model fit (AIC) compared to more complex models (Table 4). Again, in the simple models, we found seasonal model estimates during the pre-breeding were significantly lower than during the post-breeding migration, although over diurnal migration over non-barriers kestrels travelled slightly faster during the pre-breeding relative to the post-breeding migration (Table 4). Sex was not significantly different in any of the models (Table 4). 
Table 4

Comparisons between simple LMMs for mean hourly speeds $(\mathrm{km} / \mathrm{h})$ when flying over barriers or nonbarriers for diurnal and nocturnal flights accounting only for the interaction effect season:sex vs. a more complex final model accounting for the effects of season:sex and weather (tailwinds, crosswinds and BLH). BLH mainly serves as a proxy for the availability and strength of thermal updrafts; thus, we only included BLH in diurnal models. Model estimates in SD units (organised from higher to lower relative importance), standard errors $( \pm S E)$ and the variation explained $\left(R^{2}\right.$ marginal $)$ are given. Significance tests performed using ANOVA Wald chi-square test. All models included individual identity (ID) as a random effect. $(*=p \leq 0.05, * *=p \leq 0.01, * \star *=p \leq 0.001)$.

\begin{tabular}{|c|c|c|c|c|c|c|}
\hline Model & Predictor & Estimate & SE & $t$ & AIC & $\mathrm{R}^{2}{ }_{\text {mar }}$ \\
\hline \multirow{4}{*}{$\begin{array}{l}\text { Diurnal migration over } \\
\text { barriers } \\
\text { Simple model (Season:Sex) } \\
(\mathrm{n}=1910 \text {, individuals }=70)\end{array}$} & Intercept & 0.44 & 0.07 & 6.38 & \multirow[t]{4}{*}{5188.7} & \multirow[t]{4}{*}{0.03} \\
\hline & \multirow{2}{*}{$\begin{array}{l}\text { Season(Pre- } \\
\text { breeding) }\end{array}$} & -0.34 & 0.06 & $-5.25^{\star \star \star}$ & & \\
\hline & & -0.02 & 0.10 & -0.21 & & \\
\hline & Season:Sex & 0.001 & 0.11 & 0.001 & & \\
\hline \multirow{5}{*}{$\begin{array}{l}\text { Complex final model } \\
\text { (Internal + Weather) }\end{array}$} & Intercept & 0.47 & 0.05 & 8.78 & \multirow[t]{5}{*}{4869.6} & \multirow[t]{5}{*}{0.18} \\
\hline & Tailwind & 0.34 & 0.02 & $16.35^{\star \star \star}$ & & \\
\hline & BLH & -0.24 & 0.02 & $-11.08^{\star \star *}$ & & \\
\hline & \multirow{2}{*}{$\begin{array}{l}\text { Season(Pre- } \\
\text { breeding) }\end{array}$} & -0.19 & 0.05 & $-3.93^{\star \star \star}$ & & \\
\hline & & -0.12 & 0.03 & $-3.73^{\star \star \star}$ & & \\
\hline \multirow{2}{*}{$\begin{array}{l}\text { Nocturnal migration over } \\
\text { barriers }\end{array}$} & Intercept & 0.99 & 0.08 & 11.91 & \multirow[t]{4}{*}{2757.1} & \multirow[t]{4}{*}{0.03} \\
\hline & Season(Pre- & -0.36 & 0.08 & $-4.14 * \star \star$ & & \\
\hline \multirow{2}{*}{$(n=1015$, individuals $=68)$} & \multirow{2}{*}{$\begin{array}{l}\text { Sex(Male) } \\
\text { Season:Sex }\end{array}$} & 0.05 & 0.12 & 0.42 & & \\
\hline & & 0.001 & 0.12 & 0.03 & & \\
\hline \multirow{3}{*}{$\begin{array}{l}\text { Complex final model } \\
\text { (Internal + Weather) }\end{array}$} & Intercept & 0.86 & 0.06 & 13.61 & \multirow[t]{3}{*}{2640.1} & \multirow[t]{3}{*}{0.14} \\
\hline & Tailwind & 0.28 & 0.02 & 12.28 *** & & \\
\hline & Crosswind & -0.14 & 0.04 & 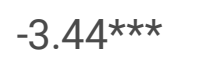 & & \\
\hline \multirow{4}{*}{$\begin{array}{l}\text { Diurnal migration over non- } \\
\text { barriers } \\
\text { Simple model (Season:Sex) } \\
(n=7328 \text {, individuals }=70)\end{array}$} & Intercept & -0.26 & 0.03 & -8.26 & \multirow[t]{4}{*}{18224.5} & \multirow[t]{4}{*}{0.01} \\
\hline & Season:Sex & -0.31 & 0.04 & $-7.55^{\star \star \star}$ & & \\
\hline & Sex(Male) & 0.12 & 0.05 & 2.42 & & \\
\hline & $\begin{array}{l}\text { Season(Pre- } \\
\text { breeding) }\end{array}$ & 0.06 & 0.02 & $2.24^{\star \star \star}$ & & \\
\hline
\end{tabular}




\begin{tabular}{|c|c|c|c|c|c|c|}
\hline Model & Predictor & Estimate & SE & $t$ & AlC & $\mathrm{R}_{\text {mar }}^{2}$ \\
\hline \multirow{7}{*}{$\begin{array}{l}\text { Complex final model } \\
\text { (Internal + Weather) }\end{array}$} & Intercept & -0.25 & 0.03 & -8.19 & \multirow[t]{7}{*}{17199.2} & \multirow[t]{7}{*}{0.15} \\
\hline & Tailwind & 0.35 & 0.01 & $32.99 * \star \star$ & & \\
\hline & Season:Sex & -0.22 & 0.03 & 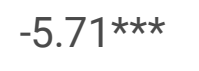 & & \\
\hline & Season (Pre- & 0.16 & 0.02 & $5.73^{\star}$ & & \\
\hline & \multirow{3}{*}{$\begin{array}{l}\text { Sex (Male) } \\
\text { Crosswind } \\
\text { BLH }\end{array}$} & 0.08 & 0.04 & 1.81 & & \\
\hline & & -0.06 & 0.01 & 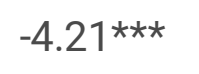 & & \\
\hline & & 0.02 & 0.01 & $2.08^{\star}$ & & \\
\hline \multirow{4}{*}{$\begin{array}{l}\text { Nocturnal migration over } \\
\text { non-barriers } \\
\text { Simple model (Season:Sex) } \\
(n=1705 \text {, individuals }=69)\end{array}$} & Intercept & 0.48 & 0.06 & 7.23 & \multirow[t]{4}{*}{5109.8} & \multirow[t]{4}{*}{0.01} \\
\hline & Season:Sex & -0.24 & 0.11 & $-2.23 *$ & & \\
\hline & Sex(Male) & 0.19 & 0.10 & 1.97 & & \\
\hline & $\begin{array}{l}\text { Season(Pre- } \\
\text { breeding) }\end{array}$ & -0.06 & 0.07 & $-0.80^{\star \star \star}$ & & \\
\hline \multirow{2}{*}{$\begin{array}{l}\text { Complex final model } \\
\text { (Internal + Weather) }\end{array}$} & Intercept & 0.46 & 0.04 & 10.89 & \multirow[t]{2}{*}{5018.1} & \multirow[t]{2}{*}{0.06} \\
\hline & Tailwind & 0.25 & 0.02 & $10.15^{\star \star \star}$ & & \\
\hline
\end{tabular}

In the final complex models, weather, in particular tailwind strength, was a strong predictor of kestrels' ground speeds (Table 4, Fig. 4). Overall, there was a considerable negative effect of absolute crosswinds on mean ground speed and a negative effect of BLH on ground speed during diurnal migration over barriers (Table 4). After accounting for wind effects, individuals displayed slight seasonal differences in mean ground speed mainly during diurnal migration (Table 4, and Supplementary Table S4). Models estimates suggested that lesser kestrels flew slower during the pre-breeding than during the post-breeding migration during diurnal flights over barriers, but also migrate faster during the pre-breeding migration during diurnal flights over non-barriers. Overall, we did not detect any strong effect of the interaction effect (season:sex), except for the model for diurnal flights over non-barriers, indicating that the speed difference between males and females during diurnal flights over non-barriers was significantly smaller during the pre-breeding relative to the post-breeding migration (Table 4).

\section{Discussion}

Variation in large-scale movement patterns (e.g. migration) is expected to be explained by the dynamic interaction among internal and external factors ${ }^{3}$. In this study, we simultaneously evaluate environmental (landscape and weather), as well as internal (sex) and other seasonal drivers in a flight generalist raptor. Flight generalists, capable of alternatively sustained flapping or soaring-gliding flight for longdistance/periods are predicted to be less affected by wind conditions compared to obligate soaring- 
gliding migratory species. Using high-resolution GPS tracking data for a large sample of individuals our study confirms considerable plasticity in lesser kestrel migration behaviour across the African-Eurasian flyways. Yet, contrary to our expectations, our work suggests that travel performance patterns were only marginally influenced by sex-specific and other seasonal drivers even when accounting for expected influences of external factors (landscape, winds) encountered by lesser kestrels along their migration routes.

In accordance with previous tracking studies on the lesser kestrel, individuals completed their migration faster during the post-breeding relative to the pre-breeding migration ${ }^{40}$. We modelled to what extend wind conditions sex-specific and other seasonal drivers could explain differences in performance. In accordance with our expectations, weather explained much of this seasonal variation, i.e. birds experienced more intense tailwinds along their realised travel direction during the post-breeding compared to the pre-breeding migration. Previous work on flight generalist birds points to the significance of tailwinds in determining speeds and travelling time, whereby prevailing winds generally opposed northward migration during the pre-breeding migration, likely causing less straight routes compared to the post-breeding migration $24,33,41$. We also found that crosswind and boundary layer height were comparatively less influential than tailwind strength on daily and hourly speeds and daily distance. Such results were expected for a flight generalist that can alternate between flapping and soaring-gliding flight to efficiently overcome crosswinds in contrast to larger birds which inevitably drift from their intended direction with every thermal ascent ${ }^{12,33}$. Although orientation behaviour (i.e. heading in relation to wind direction), is still to be investigated, kestrels seem rather prone to drift in strong winds especially over the desert $^{33}$.

In some of our models, season and the interaction between season and sex did have an effect on performance when accounting for wind effects. More specifically, kestrels appear to travel slightly faster in particular segments along their route during the pre-breeding vs. the post-breeding migration (i.e. during diurnal migration over non-barriers). We envisage two non-mutually exclusive mechanisms: (1) other external factors that we did not measure directly in this study, such as seasonality in prey availability, may favour different foraging strategies at different times of the year and (2) lesser kestrels may accelerate flight when approaching to breeding grounds. During post-breeding migration, as birds depart to their non-breeding areas in the Sahel from the Iberian and Italian peninsula, they initially travel fast due to overall shortage of food and unsuitable habitats in North Africa, and even faster in the hostile desert climate of the Sahara ${ }^{10}$. Birds are likely to engage in fly-and-forage to maximise overall migration speed, yet reducing the number of stopovers and daily distance due to foraging activity ${ }^{27}$. During the prebreeding migration, as kestrels leave non-breeding areas, they must progress fast to cross the Sahara. When they reach the north of Africa, conditions are favourable for refuelling and resting compared to post-breeding migration, as a result of winter rains ${ }^{42,43}$. Once birds have recovered at those stopover sites, birds may maximise travel speed again towards the end of their migration if they have the urgency to arrive early to secure breeding areas ${ }^{8}$. However as discussed above, seasonal and sex-specific drivers explained only little variation in travel performance in addition to weather and landscape, and it may still 
be that when considering the overall journey it is more valuable for lesser kestrels to arrive in good body condition, rather than exhausting energy reserves to ensure an early arrival.

Counter to our second prediction of faster pre-breeding migration, particularly by males, overall we did not find any sex differences in migratory performance. However, we found that after accounting for weather effects, during the pre-breeding migration males flew slower than females during diurnal migration over non-barriers relative to the post-breeding migration (Table 4). Again, these differences explained relatively little variation in performance compared to weather and landscape effects. It is important to consider that lesser kestrels reach their breeding grounds on average two months prior to the onset of breeding ${ }^{44}$. Indeed, such a long establishment phase may well offset the need for early arrivals in pre-breeding migration, and favour individuals that arrive in good condition to secure territories and prepare for reproduction ${ }^{45}$.

In agreement with our third prediction, lesser kestrels exhibited a propensity for sprinting when crossing the Sahara Desert or the Mediterranean Sea by travelling through the night as well as the day. When crossing barriers, birds thus showed a clear time-minimising behaviour in both seasons. This is also reflected in the relatively high mean hourly speeds they achieved over barriers during nocturnal flights, almost twofold the speed over non-barriers during diurnal flights (Table S3.a). In addition to the aforementioned differences in foraging propensity, these differences are most likely also influenced by variation in flight-modes, as has also been suggested for other flight generalists (gulls ${ }^{23}$, falcons ${ }^{28}$ and harriers ${ }^{24}$ ). Thermal-soaring flight is thereby expected to dominate during diurnal migration ${ }^{28}$ and flapping flight does it during nocturnal migration, although kestrels may also resort to flapping flight during the day to reduce the time needed to cross inhospitable barriers 29,46 .

We conclude that lesser kestrel exhibited great behavioural plasticity on migration, sprinting through the night across barriers, and engaging in fly-forage behaviour elsewhere. In all cases, however, tailwind assistance significantly increases the hourly and daily speed of migration, and this accounts for the faster post-breeding than pre-breeding migration in this species. We suggest a long establishments phase likely buffers against an internal motivation for faster pre-breeding migration in both sexes of the lesser kestrel. Our study generally emphasises the importance of accounting for external factors in the study of migratory performance patterns, and that seasonal and sex-specific drivers play a limited role in modulating travel performance even in flight generalist migrants.

\section{Methods}

\section{Study species and data collection}

The lesser kestrel is a small insectivorous raptor with a reversed sexual size dimorphism (females being $\sim 15 \%$ larger in body mass) ${ }^{47}$. It breeds in colonies across southern Europe, northern Africa to China and winters in Africa south of the Sahel and up to South Africa, although some Mediterranean populations also contain resident individuals. From 2014 to 2019, we trapped adult lesser kestrels at 33 breeding 
colonies in Spain and Italy and fitted them with different solar GPS-UHF biologgers (Pica, Ecotone, Gdynia, Poland; Microsensory LS, Córdoba, Spain; and NanoFix GEO + RF, Pathtrack Ltd., Leeds, UK., weighing $4-5 \mathrm{~g}$ ) attached as backpacks with a teflon harness. Loggers plus harness did not exceed the $4 \%$ of the average lesser kestrel's weight, which is within the accepted standards for animal welfare in research ${ }^{48}$. Loggers were programmed with different schedules (i.e. device's duty cycle varied from $8 \mathrm{~h}$ to $24 \mathrm{~h}$ ) and recorded GPS-locations day and night ( $65 \%$ of the tags had $24 \mathrm{~h}$ duty cycle). Over the whole migration, tracks were sampled with GPS fixes every $30 \mathrm{~min}$ to $1 \mathrm{~h}$, depending on solar battery recharge and satellite geometry ( $\geq 4$ satellites must be detected for a reliable fix). Data were stored on-board the device and downloaded the following year from successfully migrating individuals that returned to the breeding area via UHF base stations placed at the vicinity of the colony.

\section{Tracking data set}

After accounting for tag malfunctions and individuals that did not return to the colony the year after tagging, we compiled data from 72 adults who completed their migration from Spain $(n=60)$ and Italy ( $n$ $=12$ ) to Africa either along the East Atlantic or the Central Mediterranean migration flyways. Data of some of these individuals, 15 from Spain and 12 from Italy, had previously been used to analyse summer and winter population connectivity ${ }^{40}$. We included in our analyses 70 adults (40 females and 30 males) that provided 75 post-breeding and 66 pre-breeding migration tracks. Two individuals from Spain with partial migration strategies, who remained in the proximity of their breeding range, were excluded from the analysis as their non-breeding movements differed substantially from all others that migrate to African non-breeding areas.

\section{Identifying migratory trips}

GPS locations were mapped and examined visually using QGIS ${ }^{49}$. We identified migration trips based on evident long directional flights towards the south (non-breeding area) or the north (breeding range). Having identified visually the segment of data, occurring between the breeding range and the Sahel, where individuals were undoubtedly migrating, we calculated the distance between the current position to the previous one using the deg.dist function in R package 'fossil' ${ }^{50}$. Because migration is an exceptional endurance period, the onset and end of migration were identified based on marked shifts in daily distance histograms (cf. ${ }^{12}$ ). For each migratory trip, we searched for a group of first and last three consecutive days with an average daily distance of at least $150 \mathrm{~km}$, preceded (if onset) or followed (if end) by a stationary phase of five consecutive days with mean daily travelled distance $<70 \mathrm{~km}\left(\mathrm{cf}^{20}\right)$. We assigned as the start of migration day to the first of the three-days set and for the end of migration day to the last of the three-days set. We confirmed those dates visually, again using QGIS ${ }^{49}$. We excluded tracks in which we could not precisely determine the onset or the end of migration due to the lack of GPS fixes (four cases during the post-breeding and thirteen cases during the pre-breeding migration).

\section{Estimating movement metrics and their scale}


At the trip scale, we defined a migratory trip as the set of data between the first position of the start of migration day and the last position of the end of migration day. We computed: (a) the migration duration as the difference in days between the migration start and end dates; (b) the trip beeline distance (i.e. the shortest orthodromic path) as the distance between the first position of the migration start and the last position of the end of migration day; (c) the cumulative distance as the sum of the successive daily travelled distances between the start and end of migration dates; (d) trip straightness index as the ratio between the trip beeline distance and the cumulative distance; (e) the number of non-travelling days by summing days with daily distance $<50 \mathrm{~km}$ (see below); (f) migratory speed was defined as the ratio between the beeline distance and the migration duration in days (excluding non-travelling days). Means are given with standard errors throughout the paper.

At the daily scale, we defined each migration day of a kestrel from sunrise to sunrise of the next day (in our dataset lesser kestrels were frequently travelling during the night), thus capturing a complete daynight cycle. Partially due to low battery power (e.g. reduced amount of solar energy that reached the GPS panels) and different working schedules (i.e. within a range of $8 \mathrm{~h}-24 \mathrm{~h}$, as outlined above), some data gaps within migratory travel days were detected. We selected only those days with a minimum of $75 \%$ of daily coverage for this analysis. This was done to avoid bias in the calculation of daily migration metrics due to significant data gaps. The number of fixes per day was $22.72 \pm 2.00$ (mean \pm SE). We computed the following movement estimators: (a) daily beeline distance (based on all data) between the first and the last position of each unit day (sunrise to sunrise); (b) the daily travelling time, which is the cumulative sum of hourly travel segments; and (c) the daily ground speed as the daily beeline distance divided by the travelling time. Travel days were defined as those in which a kestrel's displacement in the direction of migration was at least $50 \mathrm{~km}^{33,51}$. Non-travelling days defined as complete days (sunrise to sunrise) in which less than $50 \mathrm{~km}$ of travelling in the direction of migration was observed, were excluded from further analysis.

At the hourly scale, all data were resampled to a 1-hour interval, allowing deviations up to 20 minutes, to maximise the number of observations. By resampling, we also avoided bias in our calculations of migratory parameters due to the variability in sampling frequencies ${ }^{12,52}$. After resampling, we analysed 31,153 hourly segments, from which 12,252 were annotated as travel segments (see below). We calculated the distance between the current point to the previous point using the approach outlined above and used the average to compute mean hourly ground speeds for each location. We classified as travelling segments those when speed was $\geq 5 \mathrm{~km} / \mathrm{h}$ as opposed to non-travelling segments (speed < $5 \mathrm{~km} / \mathrm{h}^{28,32}$ ). Except when indicated otherwise, hourly analyses were conducted on travelling segments.

\section{Annotating environmental variables: barriers/non-barriers, diurnal/nocturnal migration, and weather effects}

At the hourly scale, to examine possible changes in migratory behaviour of birds inside and outside barriers, we first identified when GPS fixes were located over ecological barriers, specifically over the Mediterranean Sea or the Sahara Desert (see Supplementary Methods for details) using the Global 
Biomes map ${ }^{53}$. We used the 'join-attribute-by-location' tool in QGIS ${ }^{49}$ to join all the tracks to the corresponding position within the Global Biomes map. We assigned each observation to two broad landscape categories, flying over the sea or desert to barriers, and flying outside these two regions to nonbarriers. To identify possible changes in the behaviour of kestrels in relation to the time of day (i.e. day and night), we used the sunrise.set function in R package 'StreamMetabolism'54. We classified as diurnal all locations detected between sunrise and sunset, and as nocturnal all locations between sunset and sunrise.

We built daily travel schedules in relation to hour of the day. For every migration day, the number of hourly segments were annotated according to two behaviours: travelling (if speed was $\geq 5 \mathrm{~km} / \mathrm{h}$ ) or nontravelling (if speed was $\leq 5 \mathrm{~km} / \mathrm{h}$ ) (cf. ${ }^{39}$ ). To account for the influence of atmospheric conditions on migratory decisions, we annotated each GPS fix with environmental data, namely boundary layer height (BLH) and wind using the Env-Data annotation service of Movebank ${ }^{55}$. Details on annotating weather data are provided in the Supplementary Methods.

At the daily scale, to examine how environmental drivers influence migratory parameters, we classified migration days as barrier and non-barrier days based on the proportion of time kestrels spent inside $(\geq$ $60 \%$ of daytime, barrier day) or outside ( $<60 \%$ of daytime, non-barrier day) a barrier. We computed the amount of diurnal and nocturnal travelling time by summing diurnal and nocturnal travel segments. As we expect distance to increase linearly with travelling time, we used those segments directly as control variables to account for differences in travelling time in the models accounting for weather effects. We also calculated the nocturnal travel fraction (nocturnal travelling hours/total travelling hours) and included it in the daily speed models that accounted for weather. We calculated mean daily tailwind, absolute crosswind and mean daily BLH by averaging across the day, using only travel segments.

\section{Statistical analysis: Trip scale}

To describe movement patterns, we tested for differences in performance parameters between seasons and sexes using univariate statistics. After testing for normality, we used parametric t-test for speed and non-parametric Wilcoxon rank-sum test for mean trip duration (days), mean number of travelling days, mean number of non-travelling days, and straightness index. Analyses were conducted in 'stats' 56 package for R. For pairwise comparisons we used Tukey's HSD tests, considering an effect to be significant if $p \leq 0.05$, and conducted with the 'emmeans' ${ }^{57}$ package.

\section{Statistical analysis: Daily and hourly scale}

To identify whether there was a significant difference in mean daily performance (i.e. speed, beeline distance, and travelling time) specifically between barriers and non-barriers and hourly performance between diurnal and nocturnal flight segments, we used Generalized Linear Mixed Models (GLMMs) with bird identity as a random effect. After visual inspection of residual plots, we fitted models with Gaussian error distribution for speed, daily beeline distance and Poisson error and log link function for daily travelling time, which is appropriate for count data ${ }^{58}$. For mixed models we used the Satterthwaite's 
method to estimate degrees of freedom using the 'ImerTest ${ }^{59} \mathrm{R}$ package. Multicollinearity was tested by calculating variance inflation factors (VIF) for all our predictors using the ' $\mathrm{car}^{\prime}{ }^{\prime} \mathrm{R} R$ package. Values of these were in all cases below 2.8. To analyse the daily travel schedules, hourly travel speeds were plotted in relation to the time of the day for each season and over barriers and non-barriers. In addition, we used the classification mentioned early to obtain the distribution of travelling and non-travelling segments all over the 24 hours of the day, which is a reasonable description of daily time schedules ${ }^{46}$. We compared the proportion of travelling and non-travelling segments between seasons, sexes, over barriers and nonbarriers using the Pearson's Chi-squared test using the 'vcd' ${ }^{61} \mathrm{R}$ package.

\section{Statistical analysis: wind and other seasonal drivers on daily and hourly performance}

At the daily and hourly scales we subset the data when flying over barriers vs. non-barriers, and specifically between diurnal and nocturnal flight segments at the hourly scale (again see Supplementary Note). We first used GLMMs (following the methods outlined above) to model diurnal and nocturnal performance when flying over barriers and non-barriers using as response variables: hourly and daily speeds, beeline distance and travelling time, accounting only for the interaction between season and sex (simple model). Second, to uncover mechanisms, we added weather variables (tailwinds, crosswinds and BLH) and proportion of diurnal and nocturnal flights (at daily scale) to our simple models. Prior to fitting the GLMMs, all continuous predictors and response variables were standardised to units of standard deviation, in order to compare the relative importance among predictor variables (i.e. relative effect size; cf. $^{62}$ ). The models fitted with standardised variables allow us to compare directly the effect sizes of the different estimates, representing the expected change of the response variable in units of SD, for every unit of SD change in one the continuous predictors (cf. ${ }^{12,63}$ ). We adopted a backward-model selection procedure, based on $p$-values with a threshold of 0.05 . We present both full and final models. We used Akaike's information criterion (AIC) to compare fit between simple and complex models and the variance explained by the fixed effects $\left(R_{\text {marginal }}\right)^{64}$ to compare the amount of variation explained between models. All the analyses were performed in 'Ime4'65 package.

\section{Ethical statement}

All experimental protocols were approved by Estación Biológica de Doñana Ethical Committee, Consejo Superior de Investigaciones Científicas Ethical Committee, and Consejería de Agricultura, Ganadería, Pesca y Desarrollo Sostenible de la Junta de Andalucía and carried out in accordance with relevant regulations approved by the Spanish Law on Animal Experimentation (RD53/2013 from $1^{\text {st }}$ February https://www.boe.es/eli/es/rd/2013/02/01/53). In Italy, procedures were approved by the regional authorities (Regione Sicilia n. 1616/2014 and Regione Puglia n. 475/20169) following the guidelines approved by the Law 157/1992 [Art.4(1) and Art 7(5)], which regulates research on wild bird species, and conducted mainly by the Italian Institute for Environmental Protection and Research (ISPRA). Capture and device deployment were carried out by experienced ornithologists only in accordance with approved 
guidelines aimed at ensuring animal welfare throughout the operations ${ }^{66}$. Handling time was kept to a minimum to reduce the potential for stress. No individual was injured during the capturing/handling procedure. When applicable, the design and reporting of the study were in accordance with ARRIVE Essential 10 international guidelines ${ }^{67}$.

\section{Declarations}

\section{Data availability}

GPS tracking data have been uploaded to Movebank (www.movebank.org); all datasets used in this study are available upon request from the corresponding author.

\section{Acknowledgements}

L. Lopez-Ricaurte has received financial support through the "La Caixa" INPhINIT Fellowship Grant for Doctoral studies at Spanish Research Centres of Excellence, "La Caixa" Banking Foundation, Barcelona, Spain. This project has received funding from the European Union's Horizon 2020 research and innovation programme under the Marie Skłodowska-Curie grant agreement No. 713673. We thank Aguilera M., Aguirre E., Álvarez E., Aycart P., Baena M., Bondì S., Carbonell F., Carrero M.A, de la Fuente S., de la Torre V., Galán M., Garcés M., González J.L., Griffin E., Hernández L., Holroyd E., Jordano D., Lazo P., Marfil C., Marín J., Martín-Barranco F.J., Mascara R., Meijide A., Moreno P., Morganti M., Ni Dhubhail D., Ordóñez C., Pomarol M., Pulpillo F.J., Ruiz P., Valverde A. and Zanca L. for their help during fieldwork and for technical support. We thank Fernández A. (LIFE project manager in Extremadura) for his support and collaboration, Vázquez M. for support during fieldwork in Doñana and Lourie E. for comments on previous versions of the manuscript. Funding for kestrel tagging was provided in Spain by Iberdrola España Foundation within the 'Migra' program of SEO/BirdLife, GREFA, Córdoba Zoo, Alcalá de Henares Municipality, and Global Nature Foundation within the LIFE Project "Steppe Farming" (LIFE15 NAT/ES/000734). In Extremadura tags were funded by LIFE project Gestión de ZEPA Urbanas en Extremadura (LIFE 15/NAT/ES/001016 "LIFE ZEPAURBAN), and in Andalucía by "KESTRELS MOVE" project (ref: CGL2016 79249 P) (AEI/FEDER, UE). We thank funding provided by MIUR (PRIN 20102011/20180-TZKHC). Logistic and technical support in Doñana, Spain, was provided by ICTS-RBD. GPStagging activity in Apulia (Southern Italy) was done within LIFE+Natura project "Un falco per amico" (LIFE11/NAT/IT000068). Finally, we thank Ciampanella D. (LIFE project manager), Lorusso M. (Comune di Altamura), Parisi F. (Comune di Gravina in Puglia), Giglio P. and Pellegrino S.C. (LIPU-BirdLife), and Frassanito A. (Parco Nazionale dell'Alta Murgia) for assistance and support.

\section{Author contributions}


L.L.R., W.M.G.V., J.H.P., D.G.S and J.B. conceived the study. L.L.R., D.G.S., A.B., S.C., J.G.C., J.D.L.P., F.G.T., J.M.D., A.O., B.R.M., D.R., M.S. and J.B contributed to fieldwork and gathered the tracking data. L.L.R., W.M.G.V. and J.H.P. analysed the data. L.L.R., W.M.G.V., J.H.P., D.G.S. and J.B. led the writing of the manuscript with significant input from J.G.C., D.R., M.S and all co-authors. J.H.P and J.B. supervised the study.

\section{Corresponding author}

Correspondence and request for materials should be address to L.L.R. and J.B.

\section{Competing interests}

The authors declare no competing interests.

\section{References}

1. Newton, I. The migration ecology of birds (Academic Press, 2008).

2. Akesson, S. \& Helm, B. Endogenous programs and flexibility in bird migration. Front. Ecol. Evol. 8, 78 (2020).

3. Nathan, R. et al. A movement ecology paradigm for unifying organismal movement research. Proc. Natl. Acad. Sci. USA 105, 19052-19059 (2008).

4. Alerstam, T. Optimal bird migration revisited. J. Ornithol. 152, 5-23 (2011).

5. Mellone, U., López-López, P., Limiñana, R., Piasevoli, G. \& Urios, V. The trans-equatorial loop migration system of Eleonora's falcon: differences in migration patterns between age classes, regions and seasons. J. Avian Biol. 44, 417-426 (2013).

6. Kokko, H. Competition for early arrival in birds. J. Anim. Ecol. 68, 940-950 (1999).

7. Sur, M. et al. Relevance of individual and environmental drivers of movement of Golden Eagles. Ibis 162, 381-399 (2020).

8. Karlsson, H., Nilsson, C., Bäckman, J. \& Alerstam, T. Nocturnal passerine migrants fly faster in prebreeding than in post-breeding: a test of the time minimisation hypothesis. Anim. Behav. 83, 87-93 (2012).

9. Kemp, M. U., Shamoun-Baranes, J., van Gasteren, H., Bouten, W. \& van Loon, E. E. Can wind help explain seasonal differences in avian migration speed? J. Avian Biol. 41, 672677, https://doi.org/10.1111/j.1600-048X.2010.05053.x (2010).

10. Shamoun-Baranes, J. et al. The effect of wind, season and latitude on the migration speed of white storks Ciconia ciconia, along the eastern migration route. J. Avian Biol. 34, 97-104 (2003).

11. Nilsson, C., Klaassen, R. H. G. \& Alerstam, T. Differences in Speed and Duration of Bird Migration between Pre-breeding and Post-breeding. Am. Nat. 181, 837-845 (2013). 
12. Vansteelant, W.M.G. et al. Regional and seasonal flight speeds of soaring migrants and the role of weather conditions at hourly and daily scales. J. Avian Biol. 46, 25-39 (2015).

13. Mueller, T., O’Hara, R. B., Converse, S. J., Urbanek, R. P. \& Fagan, W. F. Social learning of migratory performance. Science 341, 999-1002 (2013).

14. Miller, T. A. et al. Limitations and mechanisms influencing the migratory performance of soaring birds. Ibis 158, 116-134 (2016).

15. Dodge, S. et al. Environmental drivers of variability in the movement ecology of turkey vultures (Cathartes aura) in North and South America. Philos. Trans. R. Soc. Lond. B Biol. Sci. 369, 14712970 (2014).

16. Nourani, E., Yamaguchi, N.M., Manda, A. \& Higuchi, H. Wind conditions facilitate the seasonal watercrossing behaviour of Oriental Honey-buzzards Pernis ptilorhynchus over the East China Sea. Ibis 158, 506-518 (2016).

17. Rus, A. I., Duerr, A.E., Miller, T.A., Belthoff, J.R., \& Katzner, T. E. Counterintuitive roles of experience and weather on migratory performance. Auk 134, 485-497 (2017).

18. Thorup, K., Alerstam, T., Hake, M. \& Kjellén, N. Bird orientation: compensation for wind drift in migrating raptors is age dependent. Proc. R. Soc. Lond. B Biol. Sci. 270, S8-S11 (2003).

19. Mellone, U., Limiñana, R., López-López, P. \& Urios, V. Regional and age-dependent differences in the effect of wind on the migratory routes of Eleonora's Falcon. Curr. Zool. 61, 428-434 (2015).

20. Rotics, S. et al. Early arrival at breeding grounds: Causes, costs and a trade off with overwintering latitude. J. Anim Ecol. 87, 1627-1638 (2018).

21. Shamoun-Baranes, J., Liechti, F. \& Vansteelant, W. M. G. Atmospheric conditions create freeways, detours and tailbacks for migrating birds. J. Comp. Physiol. A. 203, 509-529 (2017).

22. Bildstein, K. L. Migrating Raptors of the World: Their Ecology and Conservation (Cornell Univ. Press, 2006).

23. Klaassen, R. H. G., Ens, B. J., Shamoun-Baranes, J., Exo, K. M. \& Bairlein, F. Migration strategy of a flight generalist, the Lesser Black-backed Gull Larus fuscus. Behav. Ecol. 23, 58-68 (2012).

24. Klaassen, R.H.G., Schlaich, A.E., Bouten, W. \& Koks, B.J. Migrating Montagu's harriers frequently interrupt daily flights in both Europe and Africa. J. Avian Biol. 48, 180-190 (2017).

25. Shamoun-Baranes, J., Bouten, W., vanLoon, E. E., Meijer, C. \& Camphuysen, C. J. Flap or soar? How a flight generalist responds to its aerial environment. Philos. Trans. R. Soc. B 371, 20150395 (2016).

26. Alerstam, T. Flight by night or day? Optimal daily timing of bird migration. J. Theor. Biol. 258, 530536 (2009).

27. Strandberg, R., \& Alerstam, T. The strategy of fly-and-forage migration, illustrated for the osprey (Pandion haliaetus). Behav Ecol Sociobiol. 61, 1865-1875 (2007).

28. Strandberg, R., Klaassen, R.H.G., Olofsson, P. \& Alerstam, T. Daily travel schedules of adult Eurasian hobbies Falco subbuteo-variability in flight hours and migration speed along the route. Ardea 97, 287-295 (2009). 
29. Hadjikyriakou, T.G., Nwankwo, E.C., Virani, M.Z. \& Kirschel, A.N. Habitat availability influences migration speed, refueling patterns and seasonal flyways of a fly-and-forage migrant. Mov. Ecol. 8, 10 (2020).

30. Hernández-Pliego, J., Rodríguez, C. \& Bustamante, J. Why Do Kestrels Soar? PLoS One 10 (2015).

31. Spaar, R. \& Bruderer, B. Migration by flapping or soaring: flight strategies of Marsh, Montagu's and Pallid Harriers in southern Israel. Condor 99, 458-469 (1997).

32. Limiñana, R., Romero, M., Mellone, U. \& Urios, V. Mapping the migratory routes and wintering areas of Lesser Kestrels Falco naumanni: new insights from satellite telemetry. Ibis 154, 389-399 (2012).

33. Limiñana, R., Romero, M., Mellone, U. \& Urios, V. Is there a different response to winds during migration between soaring and flapping raptors? An example with the Montagu's harrier and the lesser kestrel. Behav. Ecol. Sociobiol. 67, 823-835 (2013).

34. Rodríguez, A., Negro, J.J., Bustamante, J., Fox, J.W. \& Afanasyev, V. Geolocators map the wintering grounds of threatened Lesser Kestrels in Africa. Divers. Distrib. 15, 1010-1016 (2009).

35. Catry, I. et al. Individual variation in migratory movements and winter behaviour of Iberian Lesser Kestrels Falco naumanni revealed by geolocators. Ibis 153, 154-164 (2011).

36. Pedersen, L., Jakobsen, N.M., Strandberg, R., Thorup, K. \& Tøttrup, A.P. Sex-specific difference in migration schedule as a precursor of protandry in a long-distance migratory bird. Sci. Nat. 106, 45 (2019).

37. Pennycuick, C. J. Modelling the flying bird. (Elsevier, 2008).

38. Morbey, Y. E. \& Ydenberg, R. C. Protandrous arrival timing to breeding areas: a review. Ecol. Lett. 4, 663-673 (2001).

39. López-López, P., Limiñana, R., Mellone, U. \& Urios, V. From the Mediterranean Sea to Madagascar: are there ecological barriers for the long-distance migrant Eleonora's falcon? Landsc. Ecol. 25, 803-813 (2010).

40. Sarà, M. et al. Broadfront migration leads to strong migratory connectivity in the lesser kestrel (Falco naumanni). J. Biogeogr. 46, 2663-2677 (2019).

41. Mellone, U., López-López, P., Limiñana, R. \& Urios, V. Weather conditions promote route flexibility during open ocean crossing in a long-distance migratory raptor. Int. J. Biometeorol. 55, 463-468 (2011).

42. Trierweiler, C. et al. Migratory connectivity and population specific migration routes in a long-distance migratory bird. Proc. R. Soc. B 281, 20132897 (2014).

43. Vansteelant, M.G, et al. Western Marsh Harriers Circus aeruginosus from nearby breeding areas migrate along comparable loops, but on contrasting schedules in the West African-Eurasian flyway. J. Avian Biol. (2020).

44. Negro, J. J., M. De la Riva, \& J. Bustamante. Patterns of winter distribution and abundance of lesser kestrels (Falco naumanni) in Spain. J. Raptor Res 25, 31 (1991). 
45. Hubner, C. E. The importance of pre-breeding areas for the arctic barnacle goose Branta leucopsis. Ardea 94, 701-713 (2006).

46. Klaassen, R. H. G., Strandberg, R., Hake, M. \& Alerstam, T. Flexibility in daily travel routines causes regional variation in bird migration speed. Behav. Ecol. Sociobiol. 62, 1427-1432 (2008).

47. Cramp, S. \& Simmons, K. E. L. The Birds of the Western Palaearctic. Vols 1-5 (Oxford University Press, 1977-1988).

48. Barron, D. G., Brawn, J. D. \& Weatherhead, P. J. Meta-analysis of transmitter effects on avian behaviour and ecology. Methods Ecol. Evol. 1, 180-187 (2010).

49. QGIS Development Team. QGIS Geographic Information System. (Open Source Geospatial Foundation Project 2020).

50. Vavrek Matthew, J. Fossil: palaeoecological and palaeogeographical analysis tools. Palaeontol. Electron. 14, 1-16 (2011).

51. Klaassen, R. H., Hake, M., Strandberg, R. \& Alerstam, T. Geographical and temporal flexibility in the response to crosswinds by migrating raptors. Proc. R. Soc. Lond. B: Biol. Sci. 278, 1339-1346 (2010).

52. Shamoun-Baranes, J., Burant, J. B., van Loon, E. E., Bouten, W. \& Camphuysen, C. J. Short distance migrants travel as far as long distance migrants in lesser black-backed gulls Larus fuscus. J. Avian Biol. 48, 49-57 (2017).

53. Olson, D. M. et al. Terrestrial ecoregions of the world: a new map of life on earth. Bioscience 51, 933938 (2001).

54. Sefick, S. Jr. Stream Metabolism-A package for calculating single station metabolism from diurnal Oxygen curves. $R$ package version 1.2 (2016).

55. Dodge, S. et al. The environmental-data automated track annotation (Env-DATA) system: linking animal tracks with environmental data. Mov. Ecol. 1, (2013).

56. $\mathrm{R}$ development core Team. R: A language and environment for statistical computing. R Foundation for Statistical Computing. Available at: http://www.r-project.org/ (2020).

57. Russell, L. emmeans: Estimated Marginal Means, aka Least-Squares Means. R package version 1.4.5 https://CRAN.R-project.org/package=emmeans (2020).

58. Zeileis, A., \& S. Jackman, S. Regression models for count data in R. Journal of Statistical Software. 27, 1-25 (2008).

59. Kuznetsova, A., Brockhof, P. B. \& Christensen, R. H. B. ImerTest: Tests in Linear Mixed Efects Models. R package version 2.0-32 (2017).

60. Fox, J. \& Weisberg, S. An R Companion to Applied Regression. (Sage, Thousand Oaks, CA, USA, 2011).

61. Meyer, D., Zeileis, A. \& Hornik, K. vcd: Visualising categorical data. R package version 1.4-4 (2017).

62. Schielzeth, H. Simple means to improve the interpretability of regression coefficients. Meth. Ecol. Evol. 1, 103-113 (2010). 
63. Eager, C.D. Standardize: Tools for Standardising Variables for Regression in R. R package version 0.2.1. Retrieved from https://CRAN.R-project.org/package=standardize (2017).

64. Nakagawa, S. \& Hanson, P.J. A general and simple method for obtaining $R^{2}$ from generalised linear mixed-effects models. Methods Ecol. Evol. 4, 133-142 (2013).

65. Bates, D., Maechler, M., Bolker, B. \& Walker, S. Fitting linear mixed-effects models using Ime4. J. Stat. Soft. 67, 1-48 (2014).

66. Whitworth, D., Newman, S. H., Mundkur, T., \& Harris, P. Wild birds and avian influenza: An introduction to applied field research and disease sampling techniques, FAO Animal Production and Health Manual, No. 5. Rome: FAO (2007).

67. Percie du Sert, N. et al. The ARRIVE guidelines 2.0: updated guidelines for reporting animal research. Br. J. Pharmacol. 40, 1769-1777 (2020).

\section{Figures}

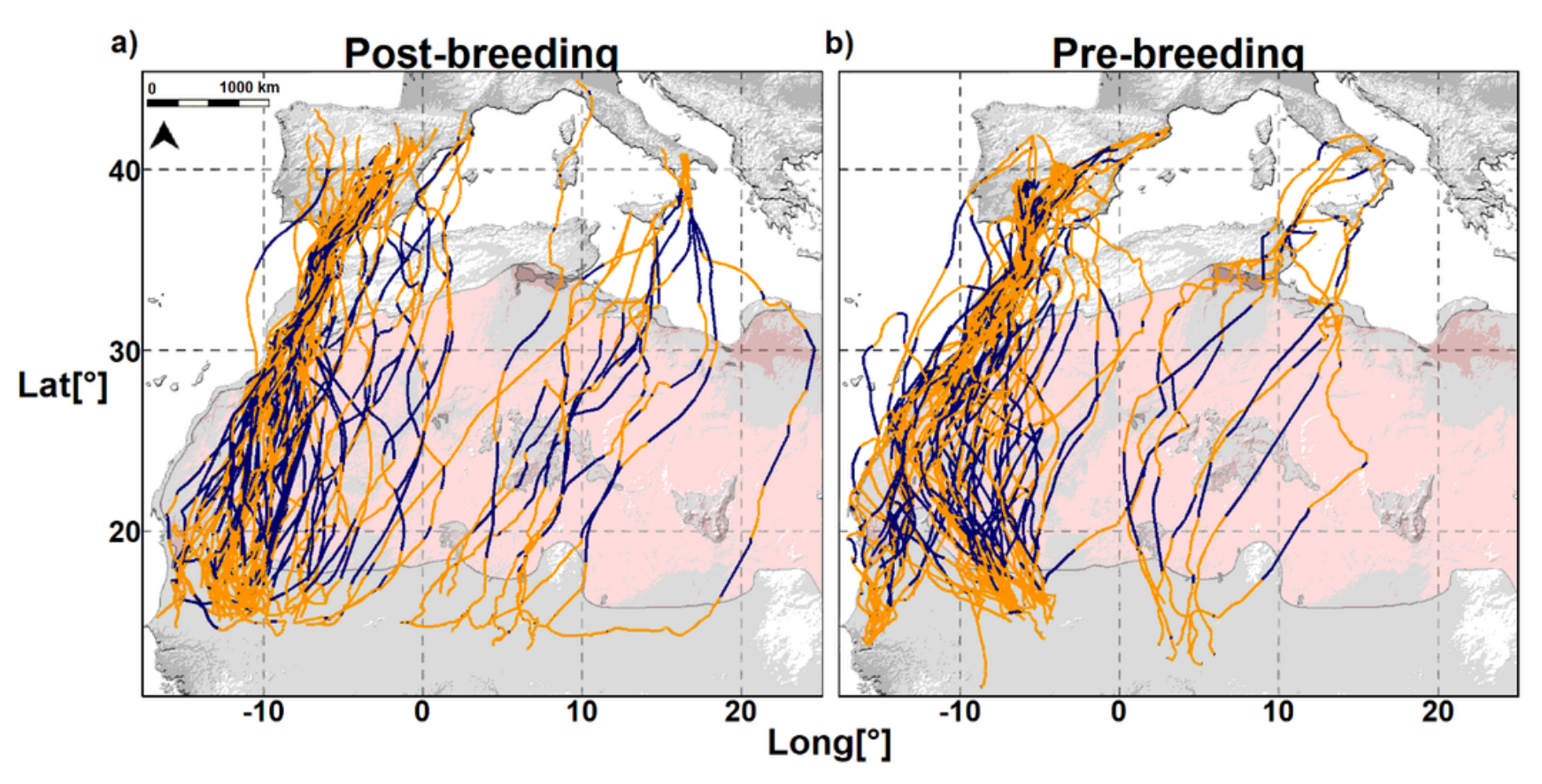


Lesser kestrel routes during the post-breeding (a) and the pre-breeding (b) migration tracked with GPS between 2014-2019. Colours indicate nocturnal migration (blue segments) and diurnal migration (orange segments) when flying over non-barriers (grey) or barriers (desert = red, sea= white). One position per hour was plotted.

a)

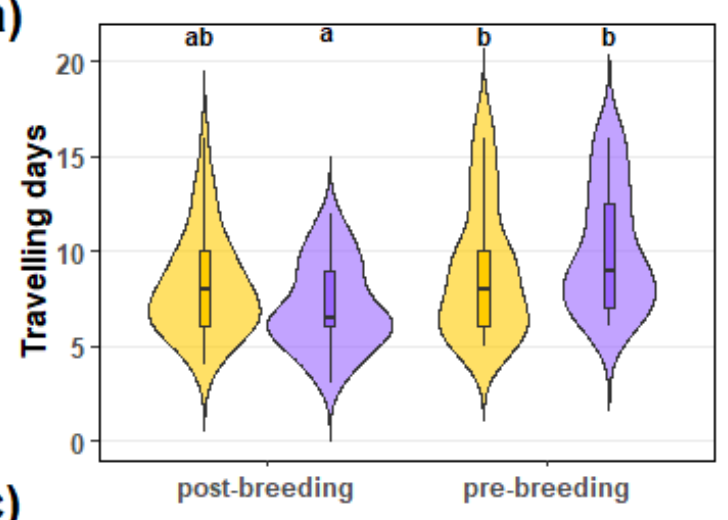

c)

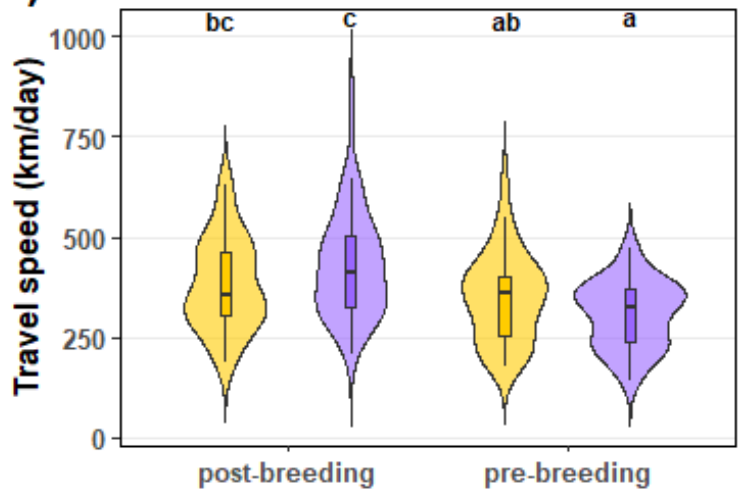

b)
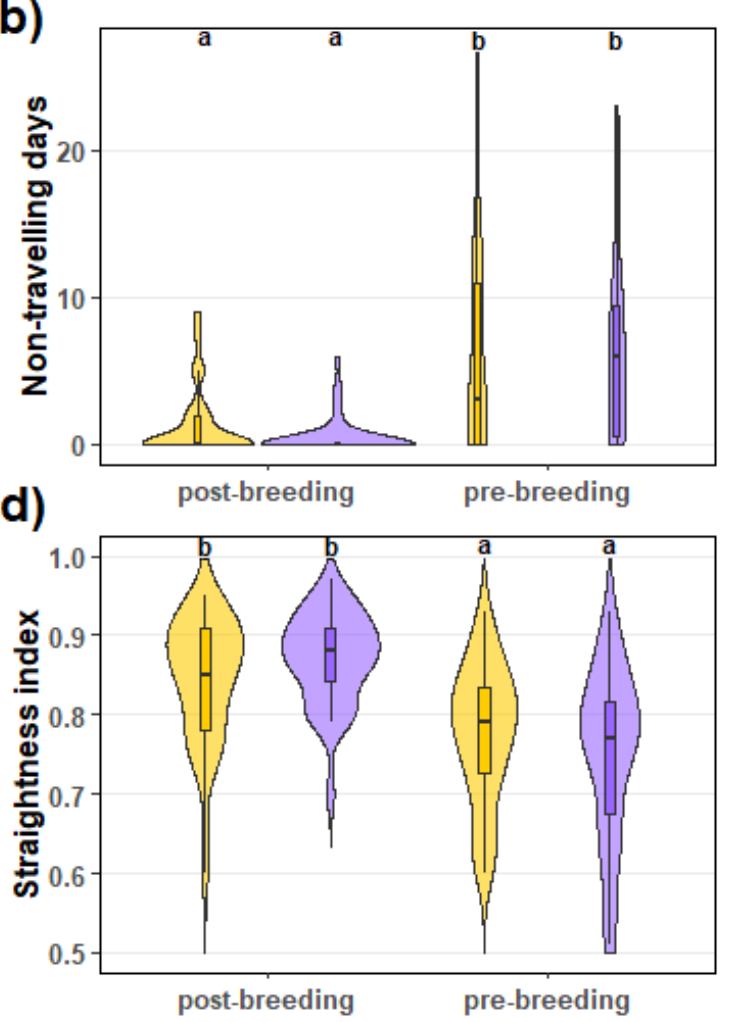

囷暒 $\mathrm{m}$

\section{Figure 2}

Violin plots showing the distribution of mean travelling days (a), mean non-travelling days (b), mean travel speed (c) and mean straightness index (d) of lesser kestrels during the post-breeding and the prebreeding migration and between sexes (females in yellow, males in purple). Comparison of means performed using Tukey's post hoc tests at the 0.05 significance level. Means sharing the same letter are not significantly different. 


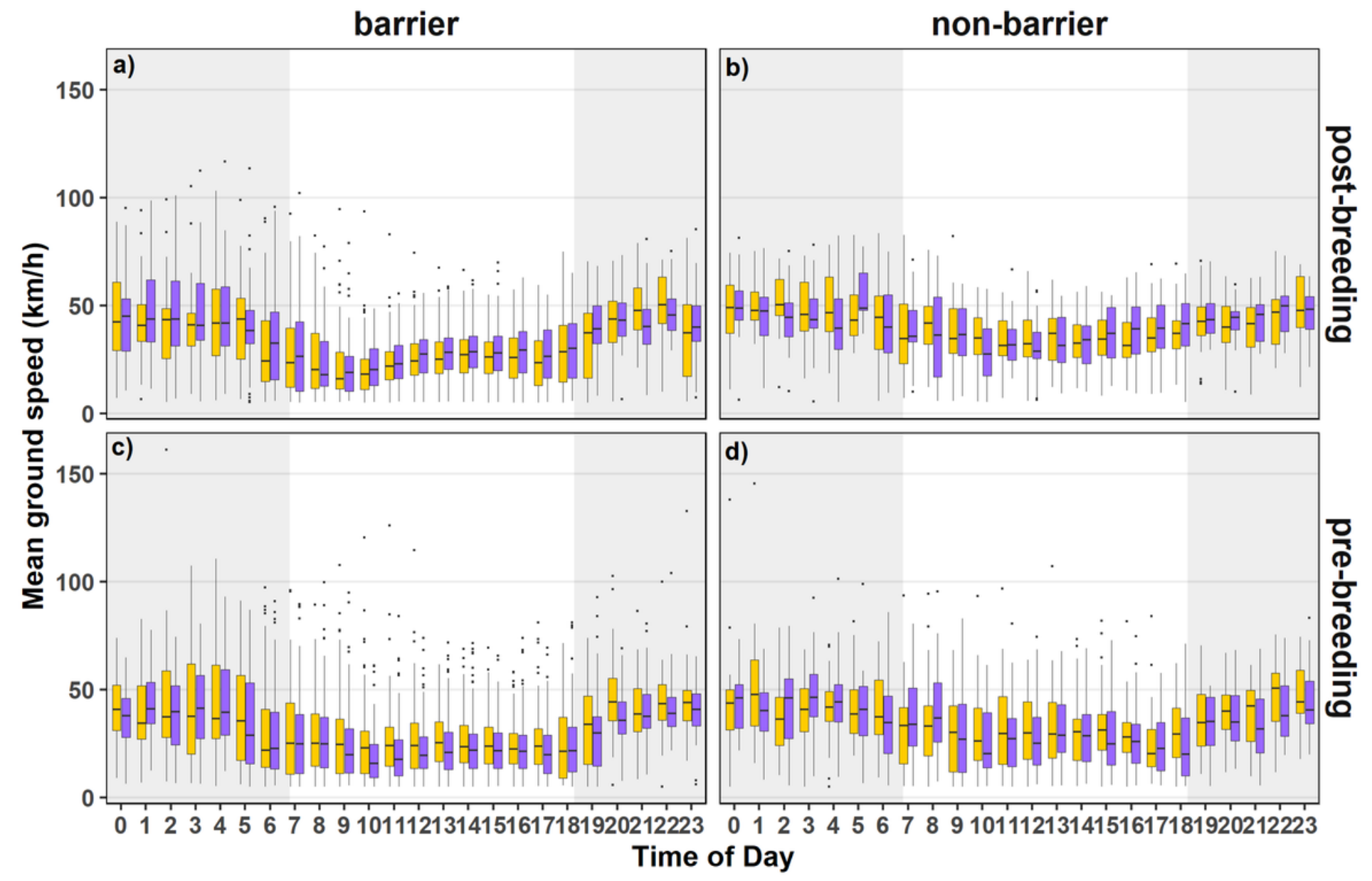

Sex

\section{Figure 3}

Boxplot for hourly mean ground speed during travelling segments for each hour of the day during the post-breeding migration over barriers (a) and non-barriers (b) and during the pre-breeding migration over barriers (c) and non-barriers (d) for each sex (females in yellow, males in purple). Grey rectangles in the background show the range of sunrise and sunset times. 


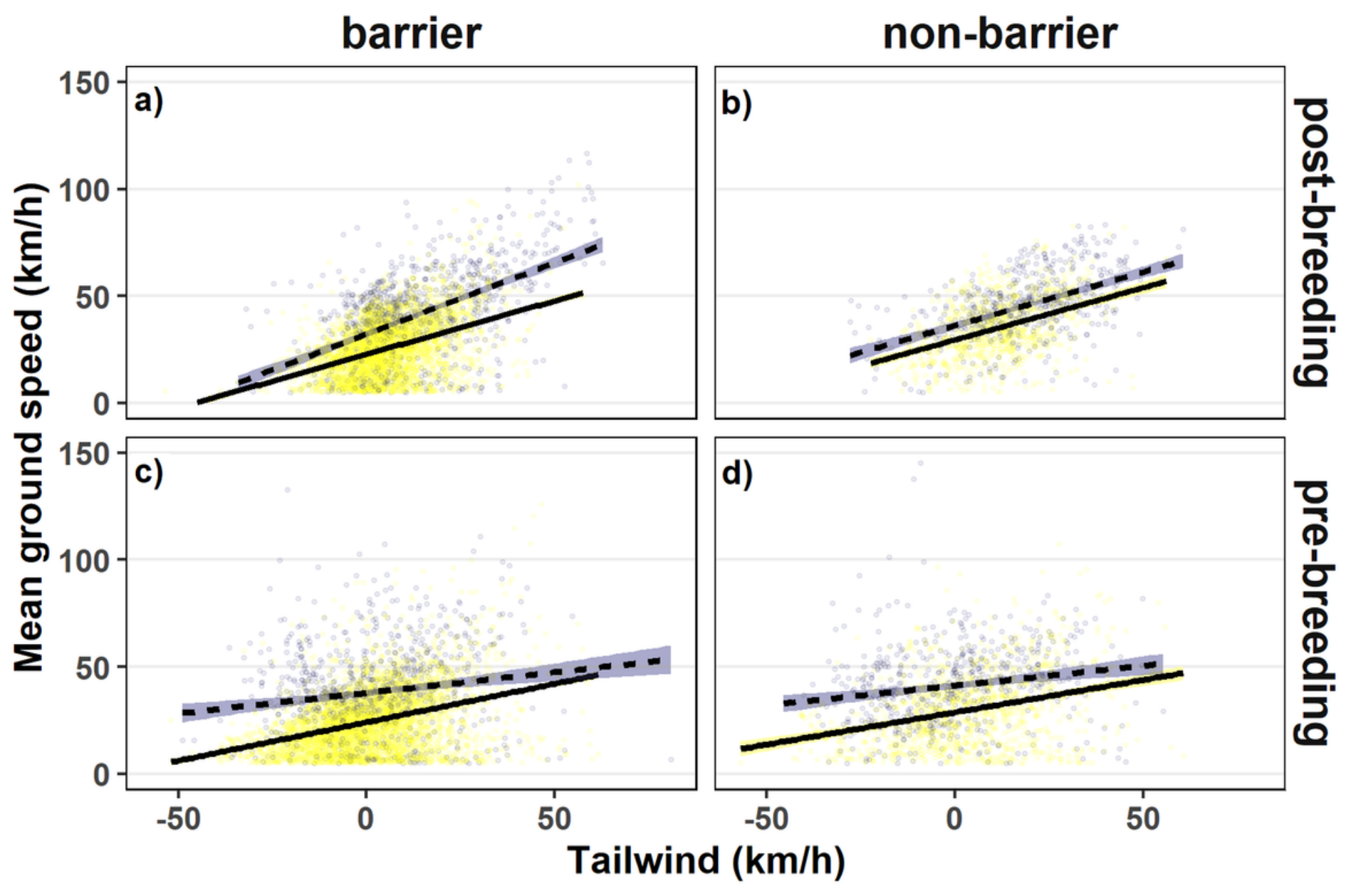

Figure 4

Relationship between average tailwinds and mean ground speed for hourly travelling segments showing the effects during the post-breeding migration over barriers (a) and non-barriers (b) and during the postbreeding migration over barriers (c) and non-barriers (d), accounting for diurnal (solid yellow line) and nocturnal migration (blue dashed line).

\section{Supplementary Files}

This is a list of supplementary files associated with this preprint. Click to download.

- Supplementarylnformation.pdf 